\title{
QoS-aware scheduling with optimization of base-station power allocation in downlink cooperative OFDMA systems
}

\author{
Xiao Zhang ${ }^{*}$, Xiaoming Tao, Yang Li and Jianhua Lu
}

\begin{abstract}
We investigate the quality of service (QoS)-aware scheduling problem for downlink cooperative orthogonal frequency division multiple access systems. Our work mainly focuses on the optimization problem for joint base-station power allocation, relay selection and subcarrier assignment with QoS guarantees. We first demonstrate that by introducing the binary assignment variables, the QoS-aware joint scheduling problem can be formulated as a mixed binary integer nonlinear programming (MBINP), which is computationally intractable. In order to solve this MBINP, power and QoS prices are introduced to transform the intractable MBINP into its corresponding dual problem via its Lagrangian, and then a two-level dual decomposition method is proposed to solve it. We derive the algorithm with the deployment of amplify-and-forward (AF) relays and/or decode-and-forward (DF) relays, analyze its computational complexity and suggest some generalizations. Finally, simulations demonstrate that our proposed method outperforms some previous works in terms of spectrum efficiency and QoS satisfaction.
\end{abstract}

Keywords: Cooperative OFDMA; Relay selection; Subcarrier assignment; Power allocation

\section{Introduction}

Cooperative relaying has recently emerged as a promising technique to realize virtual spatial diversity [1,2]. It exploits the inherent broadcasting nature of the wireless radio waves and allows distributed nodes to cooperate. This powerful technique has the potential to further enhance the overall system performance, e.g., system throughput, transmission reliability, coverage, power saving and interference mitigation. Combined with orthogonal frequency division multiple access (OFDMA), cooperative OFDMA is a strong candidate technology for modern communication systems.

To achieve full benefit of the systems (e.g., time-division multiplexing (TDM), frequency-division multiplexing (FDM), orthogonal frequency-division multiplexing (OFDM) and OFDMA and make full use of the resources (e.g., power, relays, subchannels), various scheduling problems in various systems have been extensively investigated in recent years [3-23]. Power allocation (PA) and

*Correspondence: z-xiao11@mails.tsinghua.edu.cn

Department of Electronic Engineering, Tsinghua University, Beijing, China, 100084 subchannel pairing with a single source node, a single relay and a single destination node are well studied in [3-6]. In [3], the authors considered power allocation and subcarrier pairing in two-hop multiple-input multiple-output (MIMO)-OFDM systems. Several power optimization strategies were proposed to maximize the system throughput under a sum power constraint; a subchannel pairing scheme was further proposed to improve the system throughput. The authors in [4] and [5] investigated a sum rate maximization problem with power allocation in cooperative OFDM systems. They derived the equivalent channel gain for both $\mathrm{AF}$ and $\mathrm{DF}$ relays and solved the power allocation problem by the classic waterfilling algorithm. Then a unified subchannel pairing scheme was proposed to enhance the throughput, which can be regarded as a generalization of the subchannel pairing method proposed in [3]. In [6], the authors formulated power allocation and subcarrier pairing discussed in [4] and [5] as a joint optimization problem and proposed to solve it via dual decomposition.

Regarding the scenario with a source node, multiple relays and multiple destination nodes, the scheduling problems become much more complicated to handle.

\section{Springer}

(c) 2013 Zhang et al:- licensee Springer. This is an Open Access article distributed under the terms of the Creative Commons Attribution License (http://creativecommons.org/licenses/by/2.0), which permits unrestricted use, distribution, and reproduction in any medium, provided the original work is properly cited. 
These problems are generally studied in [7-15]. In these works, quality of service (QoS) guarantees are not considered. In [7], the authors considered relay selection and subcarrier assignment for cooperative OFDMA systems. They took the fairness constraints into their model and solve the throughput maximization problem optimally by an algorithm from graph theory. The authors in [8] considered the scheduling problem in cooperative OFDMA bidirectional cellular networks. They formulated a combinatorial optimization problem using the graph theory and proposed a heuristic algorithm to jointly optimize subcarrier assignment, transmission mode selection and relay selection for the system total throughput maximization. These two graph theory-based algorithms have the limitation that they cannot perform the optimization of power allocation. The authors in [9] incorporated power allocation into the model and studied the scheduling problem for system utility maximization; a dual decomposition algorithm was proposed to the problem. The optimality of the obtained solution is guaranteed by the time-sharing condition established in [10,11].

In this work, we study QoS-aware scheduling for cooperative OFDMA systems with a source node, multiple relays and multiple destination nodes. This topic has been investigated in [16-20]. In [16], QoS-aware scheduling for relay-assisted OFDMA networks was discussed with the deployment of DF relays. By relaxing the binary assignment variables, a joint optimization algorithm was proposed to solve the relaxation problem. In [17], a power minimization problem for relay selection, and subcarrier assignment problem was formulated with consideration of QoS and fairness constraints, and a three-stage algorithm was proposed to solve it. In [18] and [19], the QoS-aware throughput maximization for relay selection and subcarrier assignment in cooperative OFDMA systems was discussed; a greedy algorithm and a dual subgradient algorithm were proposed, respectively. However, the works in [18] and [19] do not take the power allocation into account. Therefore, in this paper, we mainly investigate QoS-aware relay selection, subcarrier assignment with optimization of base station power allocation for cooperative OFDMA systems. Unlike the relaxation method adopted in [16], we treat the problem by Lagrangian relaxation and decomposition. Towards this end, we first demonstrate that by introducing binary integer variables, the complicated scheduling problem can be formulated as an MBINP, which is difficult to solve. By introducing power and QoS prices, the intractable MBINP is transformed into its corresponding dual problem via its Lagrangian, and a two-level dual decomposition method is proposed to solve it. We derive the algorithm with the deployment of AF relays and/or DF relays, analyze its computational complexity and suggest some generalizations to perform separate optimization of relay power allocation. Simulation results reveal that our proposed algorithm outperforms previous works in terms of both throughput and QoS satisfaction.

The remainder of this paper is organized as follows: in section 2, we describe the cooperative OFDMA system model and formulate the QoS-aware scheduling problem. A two-level dual decomposition algorithm is proposed to tackle the problem in section 3. Section 4 illustrates the effectiveness of the algorithms by numerical simulations. Finally, conclusions are drawn in section 5 .

\section{System model and problem formulation}

Consider a broadband wireless cellular network consisting of one base station (BS), multiple relay stations (RSs) and multiple mobile stations (MSs), each equipped with a single antenna. The sets containing $K$ RSs and $M$ MSs are denoted as $\mathcal{R}=\left\{r_{1}, r_{2}, \ldots, r_{K}\right\}$ and $\mathcal{M}=$ $\left\{m_{1}, m_{2}, \ldots, m_{M}\right\}$, respectively. All stations in the cell share a total number of $N$ subcarriers. For subcarrier $n$, the channel gains between the BS and MS $m$, the BS and RS $k$, and RS $k$ and MS $m$ are denoted as $h_{d, m}^{n}, h_{a, k}^{n}$ and $h_{b, k m}^{n}$, respectively (Figure 1 ). We assume that full channel state information (CSI) is known perfectly at the BS where the scheduling algorithm is implemented.

\subsection{Achievable rate}

The BS establishes a radio link with the MS either in cooperative or non-cooperative mode. We assume that the BS operates under time-division-duplex (TDD) mode.

\subsubsection{Direct transmission}

When the BS operates in non-cooperative mode, it communicates with the destination MS directly over two time slots. Assume the BS transmits data streams with power $P_{s, 0 m}^{n}$ to MS $m$ at subcarrier $n$ over two time slots, the instantaneous rate for the direct transmission can be written as

$$
\begin{aligned}
c_{0 m}^{n} & =\log \left(1+\mathrm{SNR}_{d, m}\right) \\
& =\log \left(1+P_{s, 0 m}^{n} d_{m}^{n}\right),
\end{aligned}
$$

where $d_{m}^{n}=\left|h_{d, m}^{n}\right|^{2} / \sigma_{m, n}^{2}$, and $\sigma_{m, n}^{2}$ represents the variance of the noise experienced by MS $m$ within the $n$th OFDMA subchannel.

\subsubsection{Cooperative transmission}

When the BS operates in cooperative mode, a proper relay should be incorporated to assist the transmission. In our discussion, we focus on two typical types of relay schemes: amplify-and-forward (AF) and decode-and-forward (DF). Assume that the BS broadcasts the data streams with 


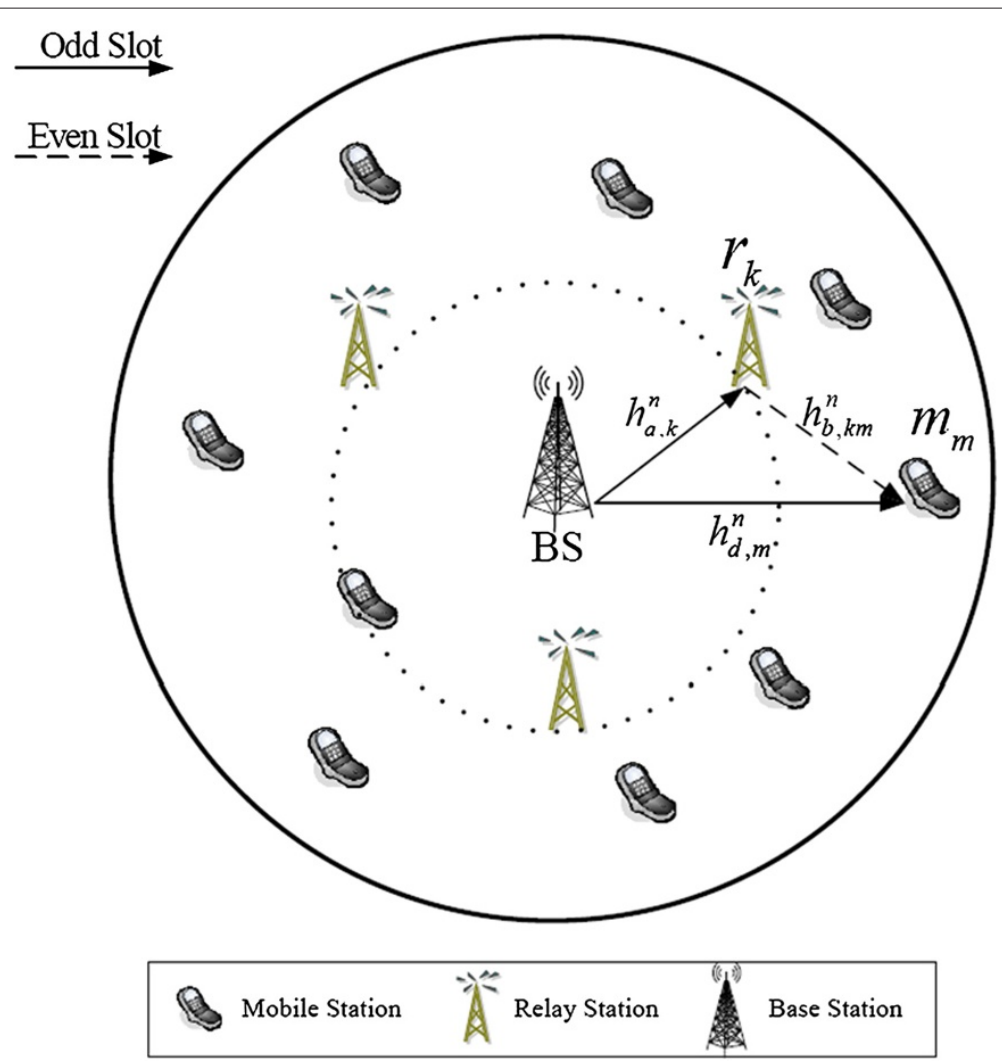

Figure 1 The system model of cooperative OFDMA networks.

power $P_{s, k m}^{n}$ to both RS $k$ and MS $m$ at subcarrier $n$ in the first time slot, then RS $k$ forwards those data streams with power $P_{r, k m}^{n}$ to MS $m$ at the same subcarrier in the second time slot. When the AF mode without a diversity protocol is adopted, the instantaneous rate of relay-mobile pair $(k, m)$ at subcarrier $n$ can be written as [1]

$$
\begin{aligned}
c_{k m, A F}^{n} & =\frac{1}{2} \log \left(1+\mathrm{SNR}_{k m, A F}^{n}\right) \\
& =\frac{1}{2} \log \left(1+\frac{P_{s, k m}^{n} a_{k}^{n} P_{r, k m}^{n} b_{k m}^{n}}{1+P_{s, k m}^{n} a_{k}^{n}+P_{r, k m}^{n} b_{k m}^{n}}\right),
\end{aligned}
$$

where $a_{k}^{n}=\left|h_{a, k}^{n}\right|^{2} / \sigma_{r, n k}^{2}, b_{b, k m}^{n}=\left|h_{b, k m}^{n}\right|^{2} / \sigma_{m, n}^{2}$, and $\sigma_{r, n k}^{2}$ denotes the variance of the noise experienced by RS $k$ within the $n$th OFDMA subchannel. For the DF scheme without a diversity protocol, the instantaneous transmission rate of relay-mobile pair $(k, m)$ at subcarrier $n$ can be written as [1]

$$
\begin{aligned}
c_{k m, D F} & =\frac{1}{2} \log \left(1+\operatorname{SNR}_{k m, D F}^{n}\right) \\
& =\frac{1}{2} \log \left(1+\min \left\{P_{s, k m}^{n} a_{k}^{n}, P_{r, k m}^{n} b_{k m}^{n}\right\}\right) .
\end{aligned}
$$

\subsection{Subcarrier constraints and the aggregate system rate} In order to formulate our scheduling problem, we first introduce the binary assignment variables $x_{\mathrm{km}}^{n}$. We define $x_{k m}^{n}=1$ indicating that the BS communicates with MS $m$ via relay $k$ at subcarrier $n$ and $x_{0 m}^{n}=1$ indicating that the BS transmits directly to MS $m$; otherwise, we have $x_{k m}^{n}=$ 0 and $x_{0 m}^{n}=0$. For the notational convenience, we denote the direct transmission link between the BS and MS $m$ as relay-mobile pair $(0, m)$. With the binary assignment variables defined, it is convenient to establish the expressions for subcarrier constraints and the aggregate system rate. The subcarrier constraints, representing the fact that each subcarrier can be occupied by one transmission link can be written as

$$
\begin{aligned}
& \sum_{k=0}^{K} \sum_{m=1}^{M} x_{k m}^{n}=1, \quad \forall n \\
& x_{k m}^{n} \in\{0,1\}, \forall k, m, n,
\end{aligned}
$$

and the aggregate system rate can also be written in a compact form as

$$
C_{\text {sum }}=\sum_{n=1}^{N} \sum_{k=0}^{K} \sum_{m=1}^{M} c_{k m}^{n} x_{k m}^{n},
$$


which is the objective function of our optimization problem.

\subsection{QoS constraints}

In this work, two types of services, namely, best-effort (BE) services and non-real-time (nRT) services are considered. $\mathrm{BE}$ services consist of applications such as e-mail and general file transfers. For BE services, the system delivers data if it must in any quantity, and therefore, $\mathrm{BE}$ services come along without any assurance of rate. $\mathrm{nRT}$ services include rate-constrained applications such as fax and voice mail. Thus, the MSs in the system can be divided into two groups. For nRT users, the rate constraints can be written as

$$
\sum_{k=0}^{K} \sum_{n=1}^{N} c_{k m}^{n} x_{k m}^{n} \geq \bar{c}_{m}, \quad m \in \mathcal{M}_{n R T},
$$

where $\bar{c}_{m}$ is the minimum rate requirement for $\mathrm{nRT}$ user $m$, and $\mathcal{M}_{n R T}$ denotes the set containing all nRT users.

\subsection{Power constraints}

In practice, the BS and RSs are operating under prescribed maximum power constraints, and the efficient allocation of power at those nodes is critical for maintaining the system throughput. In our problem, we first assume that the relay power allocation is pre-determined, and perform the optimization of base-station power allocation, and later in our discussion, we generalize our method to perform the separate optimization of relay power allocation. Thus, the power constraints at the BS are written as

$$
\begin{gathered}
\sum_{m=1}^{M} \sum_{n=1}^{N} \sum_{k=0}^{K} P_{s, k m}^{n} \leq \bar{P}_{s} \\
P_{s, k m}^{n} \geq 0, \quad \forall k, n, m,
\end{gathered}
$$

where $P_{s, k m}^{n}$ represents the power allocated at subcarrier $n$ for relay-mobile pair $(k, m)$ and $\bar{P}_{s}$ denotes the prescribed maximum allowable power at the BS.

\subsection{Optimization problem}

With all preliminaries introduced, the optimization problem for joint BS power allocation, relay selection and subcarrier assignment in downlink cooperative OFDMA networks can be formulated as follows:

$$
\begin{aligned}
\max _{X, P_{s}} & \sum_{n=1}^{N} \sum_{k=0}^{K} \sum_{m=1}^{M} c_{k m}^{n} x_{k m}^{n} \\
\text { s.t. } & \sum_{k=1}^{K} \sum_{m=1}^{M} x_{k m}^{n}=1, \quad \forall n \\
& x_{k m}^{n} \in\{0,1\}, \quad \forall k, m, n
\end{aligned}
$$

$$
\begin{aligned}
& \sum_{n=1}^{N} \sum_{k=0}^{K} c_{k m}^{n} x_{k m}^{n} \geq \bar{c}_{m}, m \in \mathcal{M}_{n R T} \\
& \sum_{m=1}^{M} \sum_{n=1}^{N} \sum_{k=0}^{K} P_{s, k m}^{n} \leq \bar{P}_{s} \\
& P_{s, k m}^{n} \geq 0, \quad \forall k, n, m \\
& c_{k m}^{n} \in\left\{c_{k m, A F}^{n}, c_{k m, D F}^{n}, c_{0 m}^{n}\right\}, \quad \forall k, m, n,
\end{aligned}
$$

where $X$ and $P_{s}$ are $(K+1) \times M \times N$ arrays consisting of all binary assignment variables $x_{k m}^{n}$ and power allocation variables $P_{s, k m}^{n}$. Note that each subcarrier can only support one relay-mobile pair, indicating $\sum_{m=1}^{M} \sum_{k=0}^{K} x_{k m}^{n}=1$. Then the constraint $\sum_{m=1}^{M} \sum_{n=1}^{N} \sum_{k=0}^{K} P_{s, k m}^{n} \leq \bar{P}_{s}$ can be equivalently replaced by $\sum_{m=1}^{M} \sum_{n=1}^{N} \sum_{k=0}^{K} P_{s, k m}^{n} x_{k m}^{n} \leq$ $\bar{P}_{s}$, resulting in

$$
\begin{aligned}
\max _{X, P_{s}} & \sum_{n=1}^{N} \sum_{k=0}^{K} \sum_{m=1}^{M} c_{k m}^{n} x_{k m}^{n} \\
\text { s.t. } & \sum_{k=1}^{K} \sum_{m=1}^{M} x_{k m}^{n}=1, \quad \forall n \\
& x_{k m}^{n} \in\{0,1\}, \quad \forall k, m, n \\
& \sum_{n=1}^{N} \sum_{k=0}^{K} c_{k m}^{n} x_{k m}^{n} \geq \bar{c}_{m}, m \in \mathcal{M}_{n R T} \\
& \sum_{m=1}^{M} \sum_{n=1}^{N} \sum_{k=0}^{K} P_{s, k m}^{n} x_{k m}^{n} \leq \bar{P}_{s} \\
& P_{s, k m}^{n} \geq 0, \quad \forall k, n, m \\
& c_{k m}^{n} \in\left\{c_{k m, A F}^{n}, c_{k m, D F}^{n}, c_{0 m}^{n}\right\}, \quad \forall k, m, n .
\end{aligned}
$$

Note that (9) is a joint optimization problem with respect to $\left(X, P_{S}\right)$. Unfortunately, it is a mixed binary integer nonlinear programming (MBINP) which is quite difficult to solve. Since both discrete binary variable $X$ and continuous variable $P_{s}$ coexist, the brute force search seems to be inapplicable. Additionally, the coupling QoS and power constraints make the problem even less tractable. In order to tackle the problem, a two-level dual decomposition method is proposed in the following section, which is demonstrated to be effective and efficient.

\section{Schedule algorithm with optimization of base-station power allocation}

In this section, we tackle the joint optimization problem (9) within the framework of the Lagrangian relaxation [24,25] and propose a two-level dual decomposition method to solve it. The general idea of our proposed algorithm is outlined briefly. First, we transform the primal problem (9) to its corresponding dual problem via 
its Lagrangian. Then, we minimize the dual problem via an inner-outer iterative algorithm. For the outer loop, the projected sub-gradient algorithm with respect to dual variables is employed to find the global minimum of the dual problem. For the inner loop, the two-level decomposition is adopted to maximize the Lagrangian for the given dual variables: in the first layer decomposition, we remove the coupling QoS and power constraints by introducing Lagrange multipliers so that the Lagrangian decouples at each subcarrier into $N$ subproblem; in the second level decomposition, by utilizing the structure of $X$, we further decompose the subproblem at each subcarrier into $M(K+1)$ subproblems. The optimal scheduling for the given dual variables can be determined by solving these $N M(K+1)$ subproblems. The optimal scheduling in the sense of minimizing the dual objective is determined when the outer projected sub-gradient algorithm converges.

\subsection{Lagrangian relaxation}

Observe that the primal problem (9) has coupling QoS and power constraints, making direct decomposition method inapplicable. Therefore, we propose to remove the coupling constraints via the Lagrangian relaxation. By introducing the Lagrange multipliers (power and QoS prices), the Lagrangian associated with (9) is derived as follows:

$$
\begin{aligned}
& L\left(X, P_{s}\right) \\
= & \sum_{n=1}^{N} \sum_{k=0}^{K} \sum_{m=1}^{M} c_{k m}^{n} x_{k m}^{n}+\sum_{m \in \mathcal{M}_{n R T}} \mu_{m}\left(\sum_{n=1}^{N} \sum_{k=0}^{K} c_{k m}^{n} x_{k m}^{n}-\bar{c}_{m}\right) \\
& +\lambda_{\mathrm{BS}}\left(\bar{P}_{s}-\sum_{m=1}^{M} \sum_{n=1}^{N} \sum_{k=0}^{K} P_{s, k m}^{n} x_{k m}^{n}\right) \\
= & \sum_{n=1}^{N}\left(\sum_{m=1}^{M} \sum_{k=0}^{K} c_{k m}^{n} x_{k m}^{n}+\sum_{m \in \mathcal{M}_{n R T}} \sum_{k=0}^{K} \mu_{m} c_{k m}^{n} x_{k m}^{n}\right. \\
& \left.-\sum_{m=1}^{M} \sum_{k=0}^{K} \lambda_{\mathrm{BS}} P_{s, k m}^{n} x_{k m}^{n}\right)-\sum_{m \in \mathcal{M}_{n R T}} \mu_{m} \bar{c}_{m}+\lambda_{\mathrm{BS}} \bar{P}_{s}
\end{aligned}
$$

where $\lambda_{\mathrm{BS}}$ is the dual variable associated with the sum power constraint for the BS and $\boldsymbol{\mu}=\left(\mu_{1}, \mu_{2}, \ldots, \mu_{\left|M_{n R T}\right|}\right)$ is the vector of dual variables for nRT service constraints, where $\left|\mathcal{M}_{n R T}\right|$ denotes the total number of $n R T$ users. Thus, the Lagrangian dual function $g\left(\lambda_{\mathrm{BS}}, \boldsymbol{\mu}\right)$ can be formulated [26]

$$
\begin{aligned}
g\left(\lambda_{\mathrm{BS}}, \boldsymbol{\mu}\right)=\max _{X, P_{s}} L\left(X, P_{s}\right) & \\
\text { s.t. } & \sum_{k=0}^{K} \sum_{m=1}^{M} x_{k m}^{n}=1, \quad \forall n \\
& x_{k m}^{n} \in\{0,1\}, \quad \forall k, m, n \\
& P_{s, k m}^{n} \geq 0, \quad \forall k, m, n \\
& c_{k m}^{n} \in\left\{c_{k m, A F}^{n}, c_{k m, D F}^{n}, c_{0 m}^{n}\right\}, \quad \forall k, m, n .
\end{aligned}
$$

The corresponding Lagrangian dual problem can be obtained [26]:

$$
\begin{array}{r}
g^{*}=\min _{\lambda_{\mathrm{BS}}, \mu} g\left(\lambda_{\mathrm{BS}}, \mu\right) \\
\text { s.t. } \lambda_{\mathrm{BS}} \geq 0, \mu \succeq 0,
\end{array}
$$

where $\succeq$ denotes the component-wise inequality for two vectors of the same length. Before solving (12), we make the following remarks.

Remark: The primal problem (9) is a MBINP, generally; strong duality does not hold, and the optimal solution obtained from minimizing (12) is near-optimal for (9). Nevertheless, due to the complex structure of problem (9), the near-optimal solution that minimizes (12) is still of great interest. Therefore, in the subsquent discussion, we aim at solving (12) to determine the near-optimal strategy for BS power allocation, relay selection and subcarrier assignment.

Via the Lagrangian relaxation, we remove the coupling QoS constraints and incorporate them into the primal objective function. We note that for fixed dual variables $\left(\lambda_{\mathrm{BS}}, \boldsymbol{\mu}\right)$, the Lagrangian $L\left(X, P_{s}\right)$ decouples at each subcarrier into $N$ independent subproblems which can be solved in parallel. The subproblem associated with subcarrier $n$ can be written as

$$
\begin{gathered}
\max _{X^{n}, P_{s}^{n}} \sum_{m=1}^{M} \sum_{k=0}^{K} c_{k m}^{n} x_{k m}^{n}+\sum_{m \in \mathcal{M}_{n R T}} \sum_{k=0}^{K} \mu_{m} c_{k m}^{n} x_{k m}^{n} \\
-\sum_{m=1}^{M} \sum_{k=0}^{K} \lambda_{\mathrm{BS}} P_{s, k m}^{n} x_{k m}^{n} \\
\text { s.t. } \sum_{k=0}^{K} \sum_{m=1}^{M} x_{k m}^{n}=1, \\
\quad x_{k m}^{n} \in\{0,1\}, \quad \forall k, m, \\
P_{s, k m}^{n} \geq 0, \quad \forall k, m, \\
c_{k m}^{n} \in\left\{c_{k m, A F}^{n}, c_{k m, D F}^{n}, c_{0 m}^{n}\right\}, \quad \forall k, m,
\end{gathered}
$$

where $X^{n}$ and $P_{s}^{n}$ are $(K+1) \times M$ matrices with elements $x_{k m}^{n}$ and $P_{s, k m}^{n}$. To simplify the notation in (13), let $\bar{\mu}_{m}=$ $\mu_{m}$, if $m \in \mathcal{M}_{n R T}$; otherwise, $\bar{\mu}_{m}=0$. Hence, (13) can be expressed in a compact form as

$$
\begin{aligned}
\max _{X^{n}, P_{s}^{n}} & \sum_{m=1}^{M} \sum_{k=0}^{K}\left(\left(1+\bar{\mu}_{m}\right) c_{k m}^{n}-\lambda_{\mathrm{BS}} P_{s, k m}^{n}\right) x_{k m}^{n} \\
\text { s.t. } & \sum_{k=0}^{K} \sum_{m=1}^{M} x_{k m}^{n}=1, \\
& x_{k m}^{n} \in\{0,1\}, \quad \forall k, m \\
& P_{s, k m}^{n} \geq 0, \quad \forall k, m \\
& c_{k m}^{n} \in\left\{c_{k m, A F}^{n}, c_{k m, D F}^{n}, c_{0 m}^{n}\right\} \quad \forall k, m, n .
\end{aligned}
$$


In order to solve (14), we introduce the following lemma.

Lemma: The optimal solution of (14) can be expressed as

$$
\left(X^{n}\right)^{*}= \begin{cases}1, & (i, j)=\arg \max _{k, m} A_{n}(k, m), \\ 0, & \text { otherwise }\end{cases}
$$

where $A_{n}$ is a $(K+1) \times M$ matrix with its element $A_{n}(k, m)$ defined as

$$
A_{n}(k, m)=\left\{\begin{aligned}
\max _{P_{s, k m}^{n}} & \left(1+\bar{\mu}_{m}\right) c_{k m}^{n}-\lambda_{\mathrm{BS}} P_{s, k m}^{n} \\
\text { s.t. } & P_{s, k m}^{n} \geq 0 \\
& c_{k m}^{n} \in\left\{c_{k m, A F}^{n}, c_{k m, D F}^{n}, c_{0 m}^{n}\right\} .
\end{aligned}\right.
$$

Observe that $X^{n}$ is a $(K+1) \times M$ matrix with only one non-zero entry. Therefore, the optimal $\left(X^{n}\right)^{*}$ that maximizing (14) can be obtained by (15). As a result, the calculation of $\left(X^{n}\right)^{*}$ can be cast as finding the maximal element in $A_{n}$. Once $A_{n}$ is determined by solving $(K+1) M$ given in (16), the optimal binary assignment matrix and allocation power matrix $\left(X, P_{s}^{n}\right)$ can be determined immediately.

In order to find the maximal element in $A_{n}$, the $(K+1) M$ subproblems given in (16) correspond to the possible $(K+1) M$ transmission links including $M$ direct links and $M \times K$ cooperative links at subcarrier $n$ should be solved. The remaining challenge is to derive the solution for each subproblem given in (16) in concrete setting.

\subsection{Solutions to individual subproblems}

In this subsection, we derive the solution to each subproblem given in (16) in concrete setting with $\left(\lambda_{\mathrm{BS}}, \boldsymbol{\mu}\right)$ fixed. We first consider the case for direct transmission and then for cooperative transmission.

\subsubsection{Direct transmission}

First, we discuss the case for direct transmission. The associated subproblem given in (16) can be written as

$$
\begin{aligned}
& \max _{P_{s, 0 m}^{n}}\left(1+\bar{\mu}_{m}\right) \log \left(1+P_{s, 0 m}^{n} d_{m}^{n}\right)-\lambda_{\mathrm{BS}} P_{s, 0 m}^{n} \\
& \text { s.t. } P_{s, 0 m}^{n} \geq 0 .
\end{aligned}
$$

Using the optimal Karush-Kuhn-Tucker (KKT) conditions [26], the closed-form solution can be obtained

$$
\left(P_{s, 0 m}^{n}\right)^{*}=\left(\frac{1+\bar{\mu}_{m}}{\lambda_{\mathrm{BS}}}-\frac{1}{d_{m}^{n}}\right)^{+}
$$

where $(y)^{+}=\max \{y, 0\}$, for $\forall y \in R$.

\subsubsection{Cooperative transmission}

For the subproblem corresponding to the AF scheme, the corresponding subproblem given in (16) can be written as

$$
\begin{aligned}
& \max _{P_{s, k m}^{n}} \frac{1}{2}\left(1+\bar{\mu}_{m}\right) \log \left(1+\frac{\alpha_{k m}^{n} \beta_{k}^{n} P_{s, k}^{n}}{1+\alpha_{k m}^{n}+\beta_{k}^{n} P_{s, k}^{n}}\right)-\lambda_{\mathrm{BS}} P_{s, k m}^{n} \\
& \text { s.t. } P_{s, k m}^{n} \geq 0
\end{aligned}
$$

where $\alpha_{k m}^{n}=P_{r, k m}^{n} b_{k m}^{n}$ and $\beta_{k}^{n}=\alpha_{k}^{n}$. By the optimal KKT conditions, the closed-form solution can also be obtained:

$$
\left(P_{s, k m}^{n}\right)^{*}=\frac{1}{\beta_{k}^{n}}\left[-1+\frac{\alpha_{k m}^{n}}{2}\left(\sqrt{1+\frac{2\left(1+\bar{\mu}_{m}\right) \beta_{k}^{n}}{\lambda_{\mathrm{BS}} \alpha_{k m}^{n}}}-1\right)\right]^{+} .
$$

For the suboptimal problem corresponding to the DF scheme, the associated subproblem given in (14) can be formulated as

$$
\begin{aligned}
& \max _{P_{s, k m}^{n}} \frac{1}{2}\left(1+\bar{\mu}_{m}\right) \log \left(1+\min \left\{P_{s, k m}^{n} a_{k}^{n}, P_{r, k m}^{n} b_{k m}^{n}\right\}\right)-\lambda \mathrm{BS} P_{s, k m}^{n} \\
& \quad \text { s.t.P } P_{s, k m}^{n} \geq 0 .
\end{aligned}
$$

Here, we define $f\left(P_{s, k m}\right)=\frac{1}{2}\left(1+\bar{\mu}_{m}\right) \log (1+$ $\left.\min \left\{P_{s, k m}^{n} a_{k}^{n}, P_{r, k m}^{n} b_{k m}^{n}\right\}\right)-\lambda_{\mathrm{BS}} P_{s, k m}^{n}$. In order to find the optimal solution of (21), we first restrict $P_{s, k m}^{n}$ to two disjoint intervals $P_{s, k m}^{n} a_{k}^{n} \geq b_{k m}^{n} P_{r, k m}^{n}$ and $P_{s, k m}^{n} a_{k}^{n} \leq$ $b_{k m}^{n} P_{r, k m}^{n}$ so that (21) is decomposed into the following two optimization problems corresponding to the two disjoint intervals:

$$
\begin{aligned}
& \max _{P_{s, k m}^{n}} \frac{1}{2}\left(1+\bar{\mu}_{m}\right) \log \left(1+b_{k m}^{n} P_{r, k m}^{n}\right)-\lambda_{\mathrm{BS}} P_{s, k m}^{n} \\
& \quad \text { s.t. } P_{s, k m}^{n} a_{k}^{n} \geq b_{k m}^{n} P_{r, k m}^{n},
\end{aligned}
$$

and

$$
\begin{aligned}
& \max _{P_{s, k m}^{n}} \frac{1}{2}\left(1+\bar{\mu}_{m}\right) \log \left(1+P_{s, k m}^{n} a_{k}^{n}\right)-\lambda_{\mathrm{BS}} P_{s, k m}^{n} \\
& \text { s.t. } b_{k m}^{n} P_{r, k m}^{n} \geq P_{s, k m}^{n} a_{k}^{n} \geq 0 .
\end{aligned}
$$

We can tackle (22) and (23) separately and then combine the optimal point of (22) and (23) to obtain the optimal solution of (21):

$$
\left(P_{s, m k}^{n}\right)^{*}=\operatorname{argmax}\left\{f\left(P_{s, m k, 1}^{n}\right), f\left(P_{s, m k, 2}^{n}\right)\right\},
$$




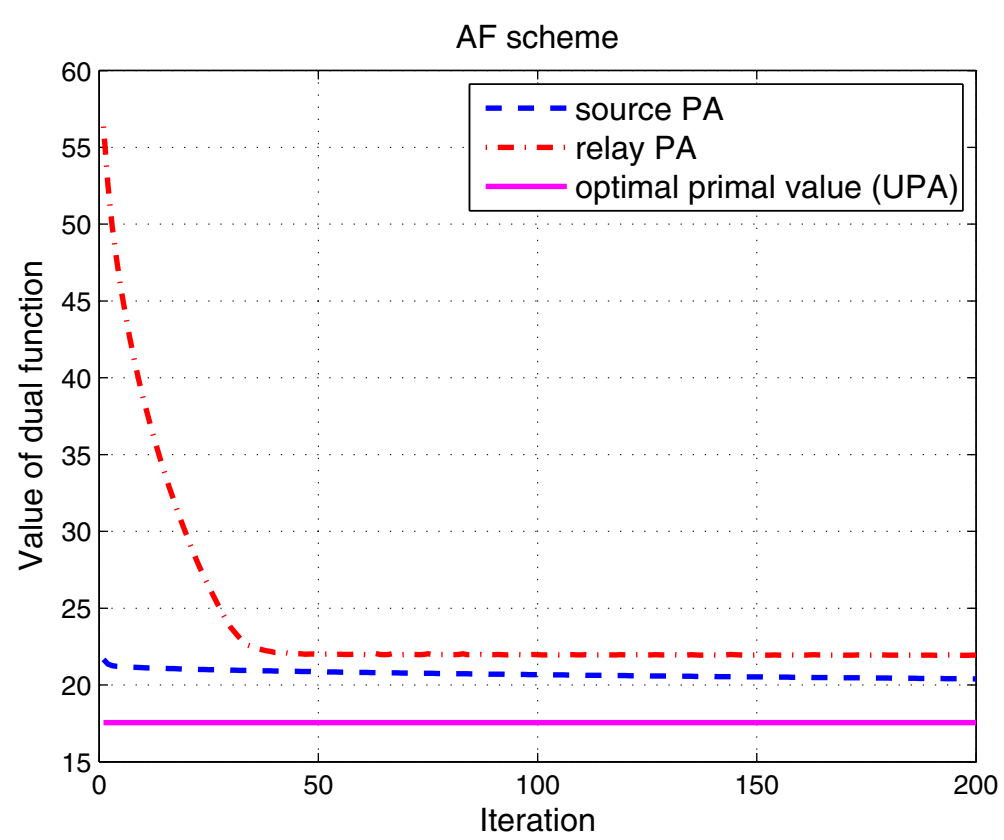

Figure 2 Dual objective value vs. iteration with AF relays, $M=6,\left|\mathcal{M}_{n R T}\right|=5, N=12$ and $\bar{c}_{1}=\bar{c}_{2}=1.5, \bar{c}_{3}=2.5, \bar{c}_{5}=2.75$, $\bar{c}_{6}=3.75$.

where $P_{s, m k, 1}^{n}=\frac{b_{k m}^{n}}{a_{k}^{n}} P_{r, k m}^{n}$ and $P_{s, m k, 2}^{n}=\mathcal{P}_{\mathcal{X}}\left(\frac{1+\bar{\mu}_{m}}{2 \lambda_{\mathrm{BS}}}-\frac{1}{a_{k}^{n}}\right)$, where $\mathcal{P}_{\mathcal{X}}$ denotes the projection onto the interval $\left[0, b_{k m}^{n} P_{r, k m}^{n} / a_{k}^{n}\right]$. We also observe that (21) can be reformulated as a convex optimization problem. By introducing the intermediate variable $t_{\mathrm{km}}^{n}$, (21) can be equivalently transformed into

$$
\begin{aligned}
& \max _{P_{s, k m}^{n}} \frac{1}{2}\left(1+\bar{\mu}_{m}\right) \log \left(1+t_{k m}^{n}\right)-\lambda_{\mathrm{BS}} P_{s, k m}^{n} \\
& \text { s.t.P } P_{s, k m}^{n} a_{k}^{n} \geq t_{k m}^{n} \\
& P_{r, k m}^{n} \geq b_{k m}^{n} t_{k m}^{n} \\
& P_{s, k m}^{n} \geq 0
\end{aligned}
$$

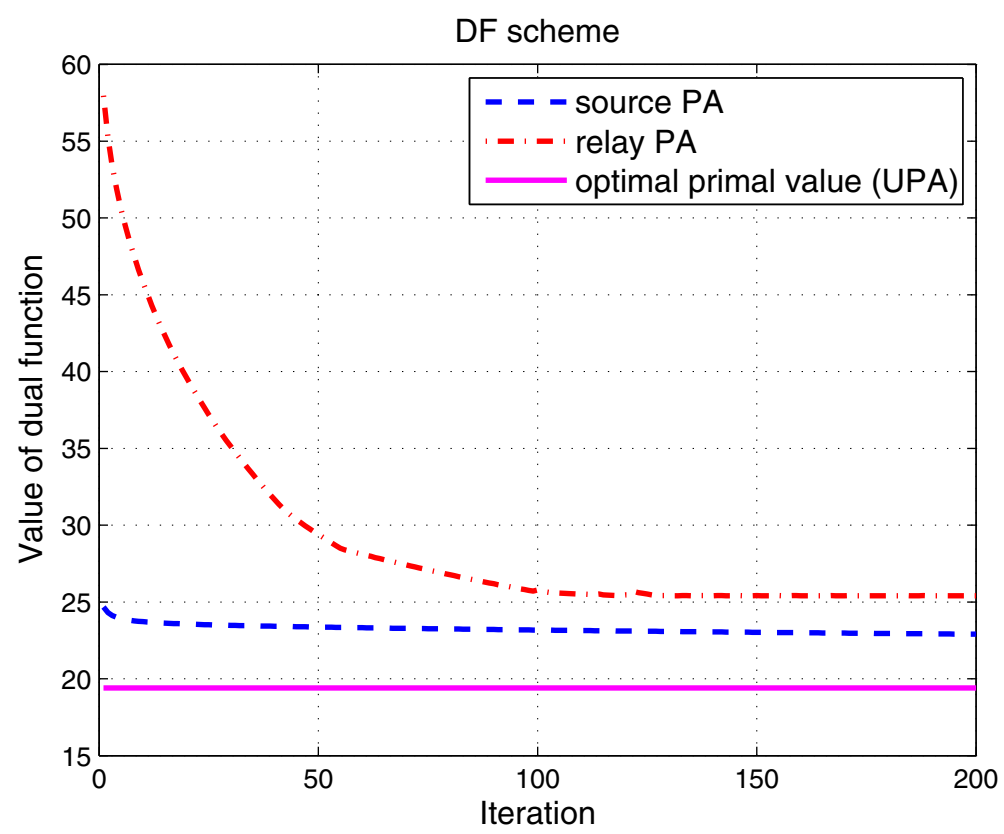

Figure 3 Dual objective value vs. iteration with DF relays, $M=6,\left|\mathcal{M}_{n R T}\right|=5, N=12$ and $\bar{c}_{1}=\bar{c}_{2}=1.5, \bar{c}_{3}=2.5, \bar{c}_{5}=2.75$, $\bar{c}_{6}=3.75$. 


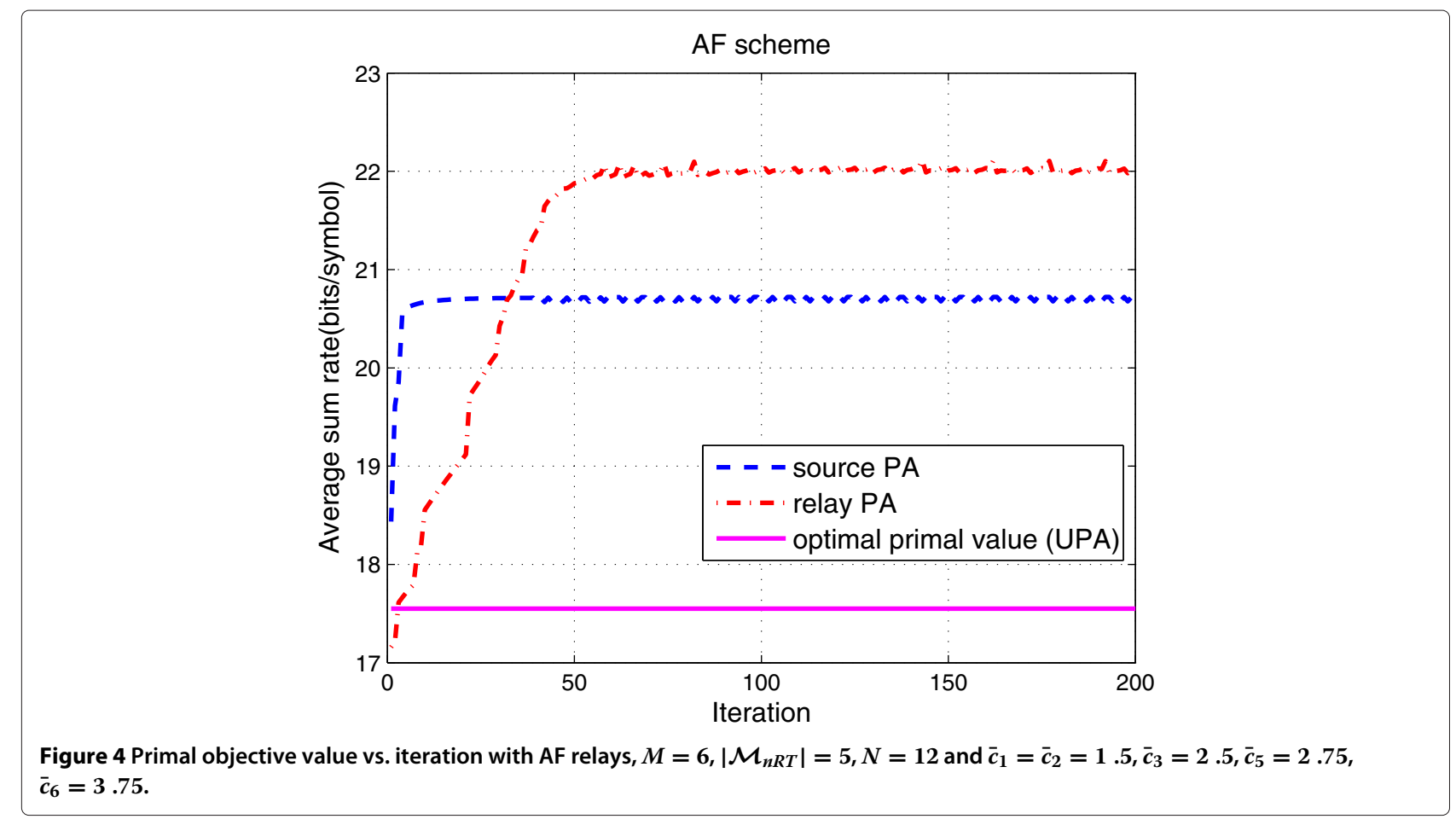

which is convex with respect to $\left(P_{s, k m}^{n}, t_{k m}^{n}\right)$. Therefore, (25) can be solved efficiently by the available CVX toolbox [27].

So far, we have derived the optimal solution of (16) with $\left(\lambda_{\mathrm{BS}}, \boldsymbol{\mu}\right)$ given, thus obtaining the optimal solution of (13). With such results available, the projected subgradient algorithm can be employed to optimize (12) in the outer loop.

\subsection{Optimization of the dual problem via projected subgradient algorithm}

By employing the projected subgradient algorithm to minimize (12), our proposed algorithm can be described as

1. Initialize $\left(\lambda_{\mathrm{BS}}^{0}, \boldsymbol{\mu}^{0}\right)$;

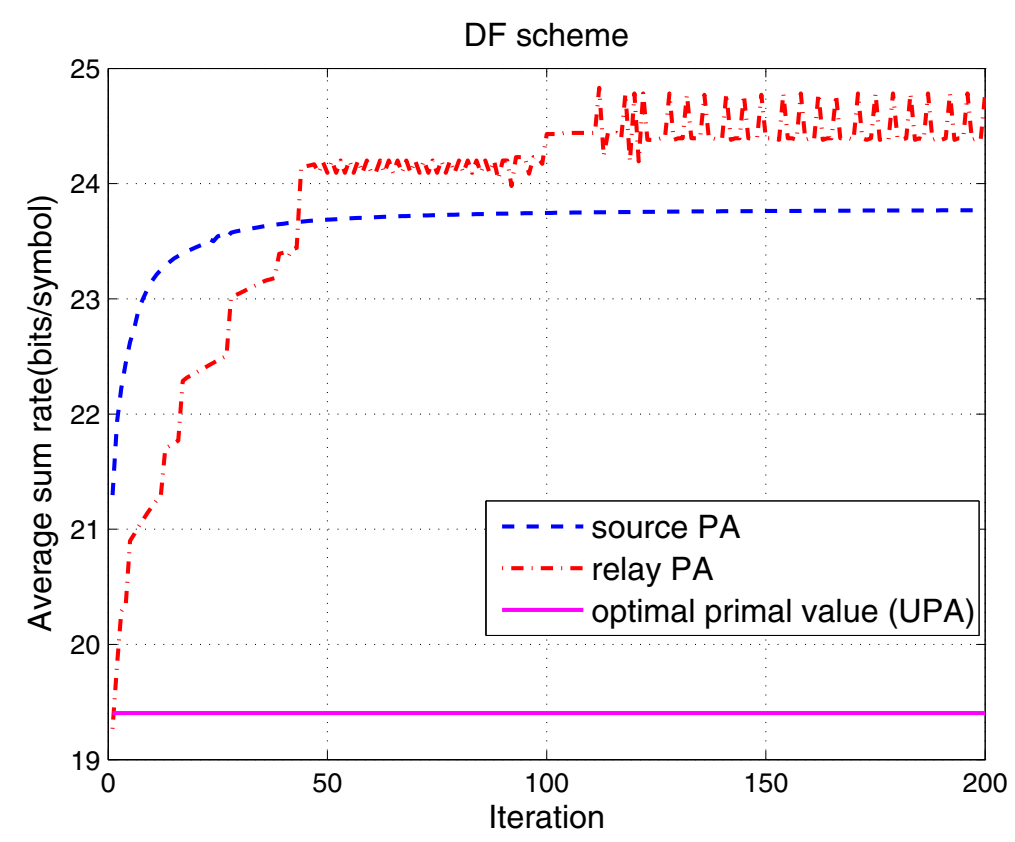

Figure 5 Primal objective value vs. iteration with DF relays, $M=6,\left|\mathcal{M}_{n R T}\right|=5, N=12$ and $\bar{c}_{1}=\bar{c}_{2}=1.5, \bar{c}_{3}=2.5, \bar{c}_{5}=2.75$, $\bar{c}_{6}=3.75$. 


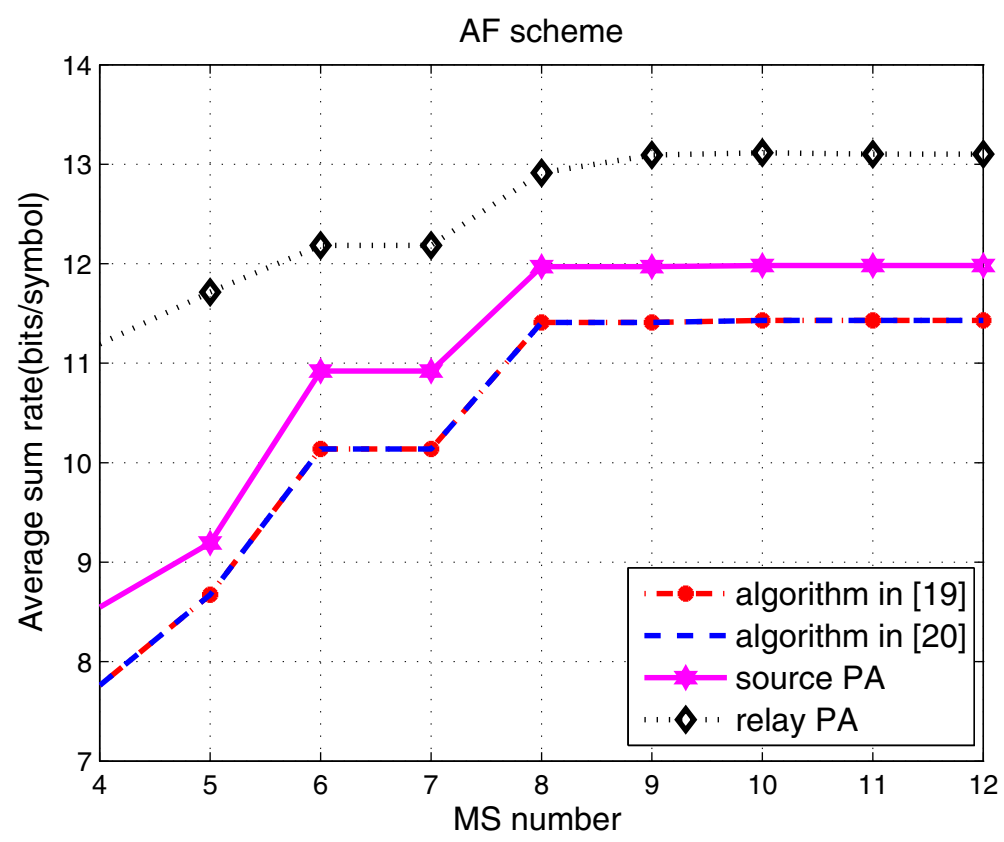

Figure 6 Average sum rate vs. MS numbers with all BE users for DF relays and $N=\mathbf{1 6}$.

2. Given $\left(\lambda_{\mathrm{BS}}^{l}, \boldsymbol{\mu}^{l}\right)$, solve $(K+1) N$ maximization problems on each subcarrier in parallel, then combine the results to obtain $\left(X_{l}^{*}(l), P_{s}^{*}(l)\right)$;

3. Perform projected subgradient updates for $\left(\lambda_{\mathrm{BS}}^{l}, \boldsymbol{\mu}^{l}\right)$

$$
\lambda_{\mathrm{BS}}^{l+1}=\lambda_{\mathrm{BS}}^{l}-s(l)\left(\bar{P}_{S}-\sum_{k=0}^{K} \sum_{n=1}^{N}\left(P_{s, k}^{n}\right)_{l}^{*}\left(x_{k m}^{n}\right)_{l}^{*}\right)^{+},
$$

$$
\mu_{m}^{l+1}=\mu_{m}^{l}-s(l)\left(\sum_{k=0}^{K} \sum_{n=1}^{N}\left(c_{k m}^{n}\right)_{l}^{*}\left(x_{k m}^{n}\right)_{l}^{*}-\bar{c}_{m}\right)^{+},
$$

$$
\forall m \in \mathcal{M}_{n R T} .
$$

4. Return to step 2 until convergence.

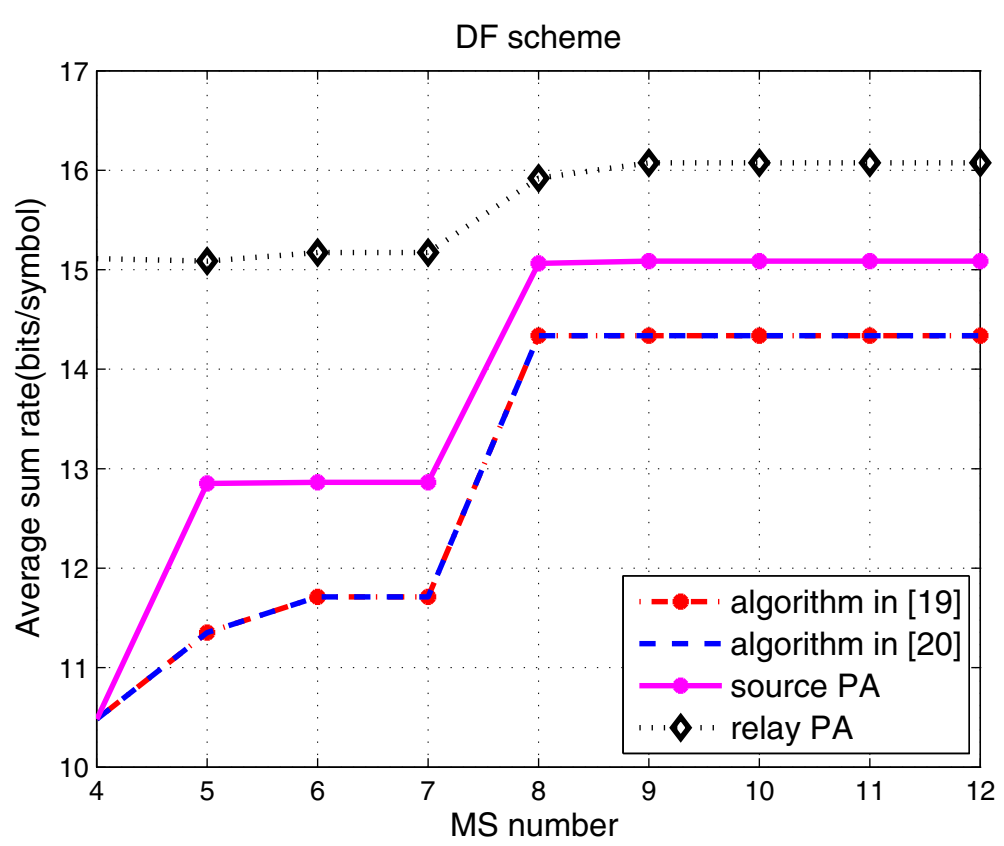

Figure 7 Average sum rate vs. MS numbers with all BE users for DF relays and $N=16$. 
Table 1 Fairness index vs. MS number with all BE users for AF relays

\begin{tabular}{|c|c|c|c|c|c|c|c|c|c|}
\hline \multirow[b]{2}{*}{ MS number } & \multicolumn{9}{|c|}{ Fairness index } \\
\hline & 4 & 5 & 6 & 7 & 8 & 9 & 10 & 11 & 12 \\
\hline Algorithm in [18] & 0.4418 & 0.4490 & 0.4484 & 0.3844 & 0.2771 & 0.2463 & 0.2283 & 0.2075 & 0.1902 \\
\hline Algorithm in [19] & 0.4418 & 0.4490 & 0.4484 & 0.3844 & 0.2771 & 0.2463 & 0.2283 & 0.2075 & 0.1902 \\
\hline Source PA & 0.5285 & 0.5937 & 0.3713 & 0.3183 & 0.2927 & 0.2601 & 0.2370 & 0.2154 & 0.1975 \\
\hline Relay PA & 0.4479 & 0.5013 & 0.6336 & 0.5431 & 0.3596 & 0.3595 & 0.3459 & 0.3190 & 0.2924 \\
\hline
\end{tabular}

For the subgradient method, if $s(l)$ in (26) satisfies the diminishing stepsize rule

$$
s(l)>0, \quad \lim _{t \rightarrow \infty} s(l)=0, \quad \sum_{l=1}^{\infty} s(l)=\infty,
$$

then

$$
\liminf _{l \rightarrow \infty} g\left(\lambda_{\mathrm{BS}}^{l}, \mu_{m}^{l}\right)=g^{*},
$$

thus obtaining a solution to the primal problem (9) accordingly.

Our optimization algorithm has an intuitive interpretation. The dual variables $\left(\lambda_{\mathrm{BS}}, \boldsymbol{\mu}\right)$ can be interpreted as the power and $Q o S$ prices and $g\left(\lambda_{\mathrm{BS}}, \mu\right)$ as its corresponding total revenue. Once the price $\left(\lambda_{\mathrm{BS}}^{l}, \mu^{l}\right)$ is set, the algorithm calculates the total revenue $g\left(\lambda_{\mathrm{BS}}, \mu\right)$ by maximizing the revenue at each subcarrier by power allocation, relay selection and subcarrier assignment. The obtained scheduling strategy obtained at the current iteration is utilized to adjust the prices used in the next iteration and the price adjustment is computed by (26). According to Lagrangian formalism, the optimal prices which minimizes (11) can be determined until the projected subgradient algorithm converges.

\subsection{Implementation issues}

We have covered nearly all details of solving the problem (12) in the previous discussion. However, as is noted, the derivation of (17), (19) and (21) is based on the implicit assumption that $\lambda_{\mathrm{BS}}$ is positive. So far, we ignore the case when $\lambda_{\mathrm{BS}}=0$ and the corresponding subproblems are unbounded above. To deal with this problem, we propose to set $P_{s, k m}^{n}$ to be $\bar{P}_{s}$, whenever $\lambda_{\mathrm{BS}}=0$, which results in an unsatisfied BS power constraint, forcing $\lambda_{\mathrm{BS}}$ to be adjusted to a positive value at the next iteration. Simulations show that the strategy works effectively.

\subsection{Complexity analysis}

In this section, computational complexity of our proposed algorithm is analyzed. For the inner loop, with fixed dual variables, all $N M(K+1)$ subproblems need to be solved to update the dual variables. For the outer loop, since the update is implemented with respect to $\left(\left|\mathcal{M}_{n R T}\right|+1\right)$ iterative variables, the complexity of the outer projected subgradient method is $O\left(\left|\mathcal{M}_{n R T}\right| / \epsilon^{2}\right)$ [28]. Therefore, the overall complexity of the algorithm for the AF and DF modes is $O\left(\left|\mathcal{M}_{n R T}\right| M N K / \epsilon^{2}\right)$. Due to the separable structure of $(10)$, the $M(K+1)$ subproblems at each subcarrier can be solved in parallel. With the CSI fed back to the BS, our proposed algorithm can be implemented efficiently.

\subsection{Generalizations}

In the previous discussion, we have focused on the problem optimizing base station power allocation with a pre-determined RS power allocation. Our method can be naturally generalized to optimize RS power allocation with a pre-determined BS power allocation. By revisiting (2) and (3)

$$
\begin{aligned}
c_{k m, A F}^{n} & =\frac{1}{2} \log \left(1+\mathrm{SNR}_{k m, A F}^{n}\right) \\
& =\frac{1}{2} \log \left(1+\frac{P_{s, k m}^{n} a_{k}^{n} P_{r, k m}^{n} b_{k m}^{n}}{1+P_{s, k m}^{n} a_{k}^{n}+P_{r, k m}^{n} b_{k m}^{n}}\right), \\
c_{k m, D F} & =\frac{1}{2} \log \left(1+\operatorname{SNR}_{k m, D F}^{n}\right) \\
& =\frac{1}{2} \log \left(1+\min \left\{P_{s, k m}^{n} a_{k}^{n}, P_{r, k m}^{n} b_{k m}^{n}\right\}\right),
\end{aligned}
$$

\begin{tabular}{|c|c|c|c|c|c|c|c|c|c|}
\hline \multirow[b]{2}{*}{ MS number } & \multicolumn{9}{|c|}{ Fairness index } \\
\hline & 4 & 5 & 6 & 7 & 8 & 9 & 10 & 11 & 12 \\
\hline Algorithm in [18] & 0.4285 & 0.3899 & 0.4301 & 0.3687 & 0.2093 & 0.1861 & 0.1675 & 0.1522 & 0.1395 \\
\hline Algorithm in [19] & 0.4285 & 0.3899 & 0.4301 & 0.3687 & 0.2093 & 0.1861 & 0.1675 & 0.1522 & 0.1395 \\
\hline Source PA & 0.4285 & 0.4156 & 0.3441 & 0.2949 & 0.2020 & 0.1806 & 0.1625 & 0.1478 & 0.1354 \\
\hline Relay PA & 0.4923 & 0.3949 & 0.3469 & 0.2973 & 0.2643 & 0.2438 & 0.2194 & 0.1995 & 0.1829 \\
\hline
\end{tabular}

Table 2 Fairness index vs. MS number with all BE users for DF relays 


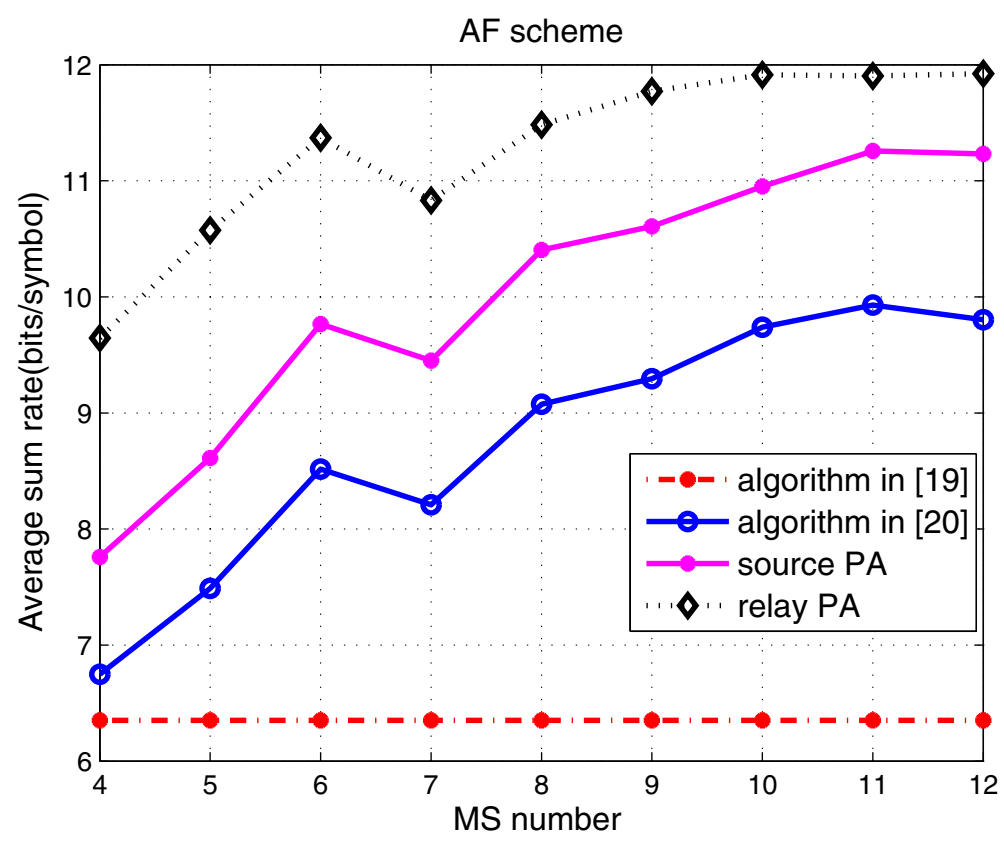

Figure 8 Satisfaction index vs. MS number with all nRT users for AF relays, $N=16$.

we note that with $P_{s, k m}^{n}$ given, the optimization can be similarly performed with respect to $P_{r, k m}^{n}$. The method proposed above can be applied with minor revisions.

\section{Simulation results}

In this section, we present simulation results that verify the performance of our proposed method. We consider a relay-aided OFDMA network with the BS located at the center of the cell with a radius of $d_{1}=1 \mathrm{~km}$. Four RSs are placed uniformly on a circle with a radius of $d_{2}=0.5 \mathrm{~km}$. All MSs are randomly located in the cell with radii between $d_{3}=0.95 \mathrm{~km}$ and $d_{4}=1 \mathrm{~km}$. The channel is modeled to experience both the largescale and small-scale fadings. By adopting Clarke's model,

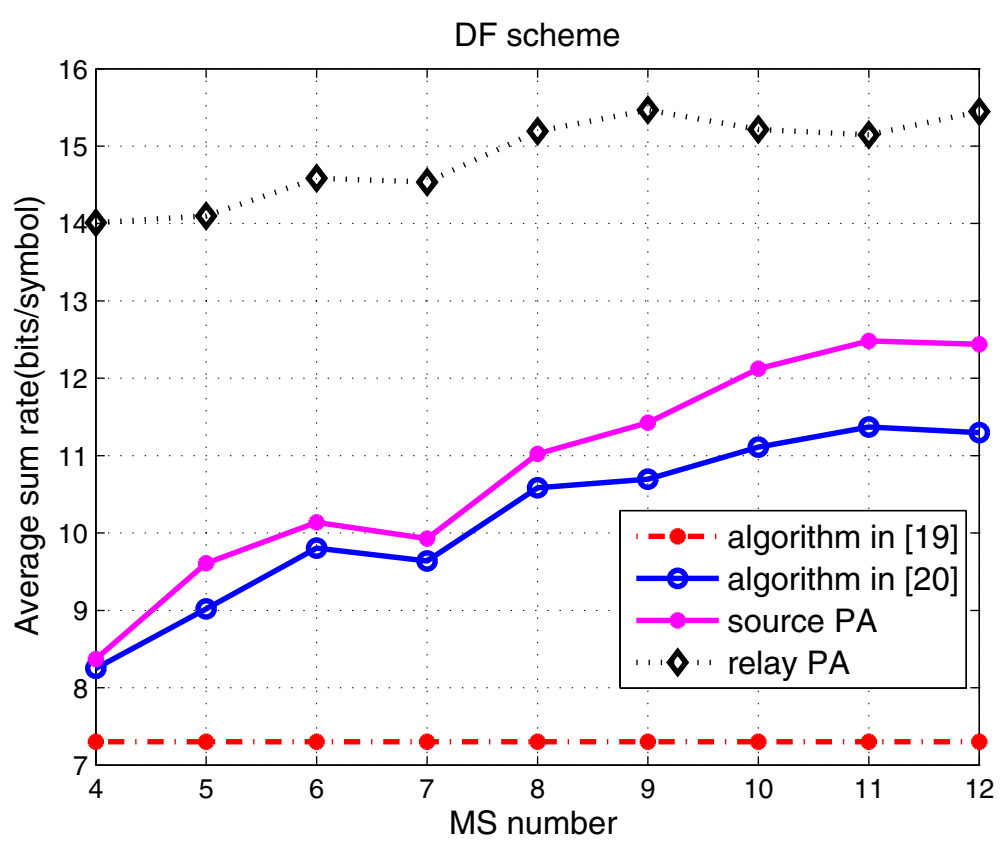

Figure 9 Average sum rate vs. MS number with all nRT users for DF relays, $N=16$. 


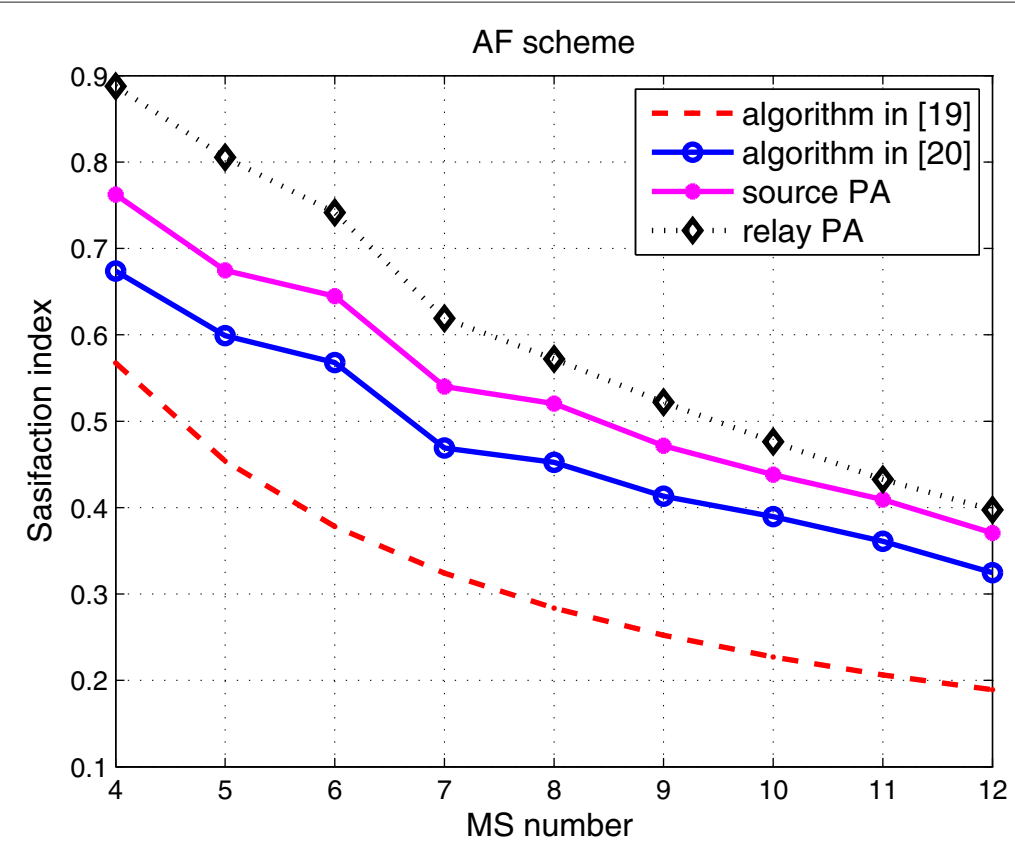

Figure 10 Satisfaction index vs. MS number with all nRT users for AF relays, $N=16$.

the frequency selective channel is simulated by six independent Rayleigh multipaths. The path loss is modeled by a modified COST231-Hata propagation model with $128+38 \log (R)$, where $R$ denotes the distance in kilometers. Further, the log-normal shadowing is assumed to be zero mean with a standard deviation of $8 \mathrm{~dB}$. The total bandwidth is $1.25 \mathrm{MHz}$ and the power spectral density of the noise is $-155 \mathrm{dBm} / \mathrm{Hz}$. In our simulation, we first demonstrate some typical convergence behaviors of our proposed method. Then we compare our proposed algorithm denoted source PA discussed in the main body of the paper and relay PA mentioned in section 3.6 with

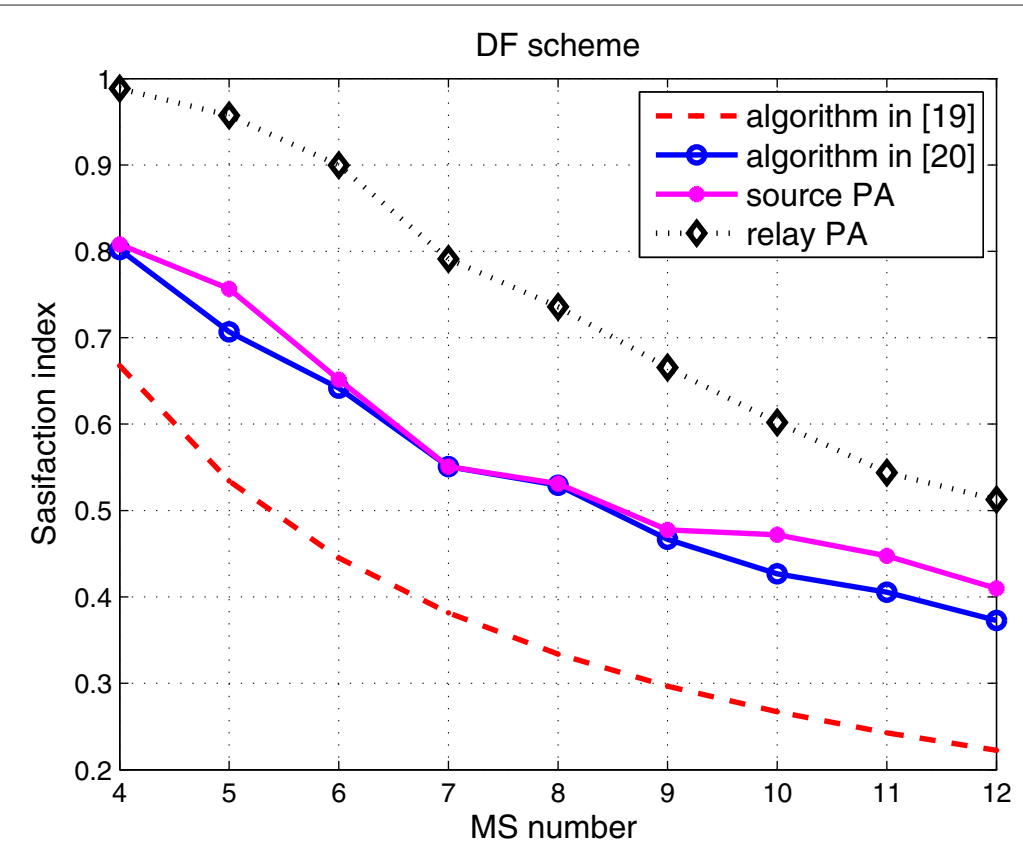

Figure 11 Satisfaction index MS number with all nRT users for DF relays, $N=16$. 
Table 3 Fairness index vs. MS number with all nRT users for AF relays

\begin{tabular}{lcccccccrr}
\hline MS number & \multicolumn{1}{c}{ Fairness index } & $\mathbf{8}$ & $\mathbf{9}$ & $\mathbf{1 0}$ & $\mathbf{1 1}$ \\
\hline Algorithm in [18] & $\mathbf{4}$ & $\mathbf{5}$ & $\mathbf{6}$ & $\mathbf{7}$ & $\mathbf{8}$ & $\mathbf{1 2}$ \\
Algorithm in [19] & 0.9083 & 0.4867 & 0.4056 & 0.3476 & 0.3042 & 0.2704 & 0.2433 & 0.2212 & 0.2028 \\
Source PA & 0.9631 & 0.9145 & 0.9155 & 0.8615 & 0.8029 & 0.7745 & 0.7937 & 0.7505 & 0.6243 \\
Relay PA & 0.9430 & 0.9454 & 0.9319 & 0.9376 & 0.9047 & 0.8496 & 0.8637 & 0.8327 & 0.7852 \\
\hline
\end{tabular}

the algorithms proposed in [18] and [19] under several performance measures. Simulations are conducted using $s(l)=A / \sqrt{l}$, where $A$ is a suitable positive scalar. For simplicity, all algorithms are implemented with one relay type (AF or DF).

In Figures 2 and 3, some typical convergence results of our proposed method are illustrated. The optimal primal value under uniform power allocation (UPA) assumption (the horizontal line) is also displayed. By weak duality, the optimal value serves as a lower bound of the dual objective value. We also observe from Figures 2 and 3 that when the initial point and step size are properly chosen, the algorithm to the dual problems converges. To demonstrate the effectiveness of our proposed algorithm, the corresponding primal objective value from each iteration is also displayed in Figures 4 and 5. By comparing the primal objective value obtained from each subgradient iteration with the optimal solution obtained under UPA assumption, we see that our proposed algorithm taking power allocation into account improves the system throughput.

After demonstrating the convergence behaviors of our proposed algorithm, we also compare our proposed algorithm with the algorithms proposed in [18] and [19] in terms of the average sum rate and average fairness index. Jain's Fairness Index is employed as a measure of average fairness [29], which is formally defined as FI $=$ $\left(\sum_{m=1}^{M} c_{m}\right)^{2} /\left(M \sum_{m=1}^{M} c_{m}^{2}\right)$, where $M$ is the total number of MSs. A scenario with all BE users is considered. We first initialize the cell with $4 \mathrm{BE}$ users, then incrementally add additional $\mathrm{BE}$ users into the cell until the total number of MSs reaches 12, performing the scheduling algorithms at each step. Figures 6 and 7 illustrate the average sum rates achieved by each algorithm with AF relays or DF relays. From Figures 6 and 7, we note that when all MSs are BE users, the algorithms proposed in [18] and [19] have the same performance. We also observe that the algorithm in [19] converges after one iteration. Additionally, under the all-BE-user assumption, the average sum rate remains non-decreasing: when one BE user with better CSI is incorporated, the system would reallocate the resources to increase the average sum rate; when one user with very poor CSI is incorporated, the system would not allocate any resources to it resulting in the non-decreasing property of the average sum rate. In Figures 6 and 7, we can infer that MS 8 is with the best CSI. Average fairness index (FI) is also displayed in Tables 1 and 2. From Tables 1 and 2, we note that with the increase of BE users, the FI tends to degrade. The phenomenon reflects the fact that the average sum criterion favors BE users with better CSI, resulting in the degradation of $\mathrm{FI}$, which conforms to the traditional point of view. We conclude that our proposed algorithm performs better in terms of the average sum rate.

A scenario with all nRT users is considered. Similar to the last simulations conducted above, we start with four $\mathrm{nRT}$ users and then place one additional nRT user into one cell at a time. All nRT users are with a minimum rate requirement of 2.5. Due to the QoS demanded by each $\mathrm{nRT}$ user, the average satisfaction index is employed as a measure of average QoS satisfaction, which is formally defined as SI $=\frac{1}{M} \sum_{m=1}^{M} \min \left(\frac{c_{m}}{\bar{c}_{m}}, 1\right)$, where $M$ is the total number of MSs. Figures 8, 9, 10 and 11 illustrate the average sum rates and average satisfaction index (SI) achieved by four algorithms with AF relay or DF relays. Opposed to Figures 6 and 7, the average sum rate displayed in Figures 8

Table 4 Fairness index vs. MS number with all nRT users for DF relays

\begin{tabular}{|c|c|c|c|c|c|c|c|c|c|}
\hline \multirow[b]{2}{*}{ MS number } & \multicolumn{9}{|c|}{ Fairness index } \\
\hline & 4 & 5 & 6 & 7 & 8 & 9 & 10 & 11 & 12 \\
\hline Algorithm in [18] & 0.7148 & 0.5718 & 0.4765 & 0.4084 & 0.3574 & 0.3177 & 0.2859 & 0.2599 & 0.2383 \\
\hline Algorithm in [19] & 0.9609 & 0.9417 & 0.9245 & 0.8849 & 0.9006 & 0.7859 & 0.6385 & 0.6558 & 0.6370 \\
\hline Source PA & 0.9706 & 0.9786 & 0.9259 & 0.8169 & 0.8329 & 0.7541 & 0.7663 & 0.7857 & 0.8060 \\
\hline Relay PA & 0.9265 & 0.9656 & 0.9417 & 0.8752 & 0.8486 & 0.8323 & 0.8351 & 0.7834 & 0.7608 \\
\hline
\end{tabular}


and 10 is no longer a non-decreasing function of the number of nRT MSs. With all AF relays, especially when MS 7 is incorporated, the average sum rate tends to decrease. This is mainly due to the poor CSI of MS 7: when one nRT MS with poor CSI is incorporated, owing to the QoS constraint imposed, the system has to allocate some resources to it, resulting in a decrease of the sum average rate. When MS 8 is incorporated, the average sum rate tends to increase. This is owing to the better CSI of MS 8: when an nRT MS with better CSI is incorporated, with limited resources, the system would allocate more resources to the user for maximizing the average sum rate. We also observe that as the number of nRT users increases, the systems cannot support all the nRT users simultaneously, leading to the decrease of SI. As the SI index decreases simultaneously, our proposed algorithm improves the SI compared with the algorithms proposed in [18] and [19]. FI is displayed in Tables 3 and 4. We conclude that with the increase of nRT users, the FI tends to decrease. Although the projected subgradient method may exhibit some fluctuations in primal value (Figures 4 and 5), we conclude that our proposed algorithm taking power allocation into account outperforms some previous works in terms of spectrum efficiency and QoS satisfaction.

\section{Conclusion}

For exploiting fully the merits of cooperative OFDMA with limited resources, we investigated the scheduling problem with the separate optimization of base station and relay power allocation for cooperative OFDMA systems where BE and nRT services are supported simultaneously. We formulated the scheduling problem as an MBNLP and proposed a two-level dual decomposition algorithm to solve it. We derive the algorithm with deployment of AF relays and/or DF relays. Simulation results demonstrate that our proposed algorithm taking power allocation into account outperforms some previous works in terms of spectrum efficiency and QoS satisfaction.

\section{Competing interests}

The authors declare that they have no competing interests.

\section{Acknowledgements}

This work was supported by the National Basic Research Project of China (973) (2013CB329001), National Natural Science Foundation of China (NSFC, No.61101071, 61132002, 61021001). This work was presented in part at the IEEE Vehicular Technology Conference, Quebec City, Canada, Sep. 2012.

Received: 30 January 2013 Accepted: 3 October 2013

Published: 23 October 2013

\section{References}

1. JN Laneman, D Tse, G Wornell, Cooperative diversity in wireless networks: efficient protocols and outage behavior. IEEE Trans. Inf. Theory 50(12), 3062-3080 (2004)

2. JN Laneman, GW Wornell, Distributed space-time coded protocols for exploiting cooperative diversity in wireless networks. IEEE Trans. Inf. Theory 49(10), 2415-2425 (2003)
3. I Hammerstrom, A Wittneben, Power allocation schemes for amplify-and-forward MIMO-OFDM relay links. IEEE Trans. Wireless Commun. 6(8), 2798-2802 (2007)

4. $Y$ Li, W Wang, J Kong, W Hong, X Zhang, M Peng, in Proc. IEEE International Conference on Communications (ICC'08). Power allocation and subcarrier pairing in OFDM-based relaying networks (Beijing, May 19-23, 2008), pp. 2602-2606

5. Y Li, W Wang, J Kong, M Peng, Subcarrier pairing for amplify and-forward and decode-and-forward OFDM relay links. IEEE Commun. Lett. 13(4), 209-211 (2009)

6. C-N Hsu, H-J Su, P-H Lin, Joint subcarrier pairing and power allocation for OFDM transmission with Decode-and-Forward relaying. IEEE Trans. Signal Process 59(1), 399-414 (2011)

7. G Li, H Liu, Resource allocation for OFDMA relay networks with fairness constraints. IEEE J. Selected Areas Commun. 24(11), 2061-2069 (2006)

8. Y Liu, M Tao, B Li, H Shen, Optimization framework andgraph-based approach for relay-assisted bidirectional OFDMA cellular networks. IEEE Trans. Wireless Commun. 9(11), 3490-3500 (2010)

9. $\mathrm{TNg}, \mathrm{W} Y \mathrm{u}$, Joint optimization of relay strategies and resource allocations in cooperative cellular networks. IEEE J. Selected Areas Commun. 25(2), 328-339 (2007)

10. W Yu, R Lui, Dual methods for nonconvex spectrum optimization of multicarrier systems. IEEE Trans. Commun. 54(7), 1310-1322 (2006)

11. Z Luo, S Zhang, Dynamic spectrum management: complexity and duality. IEEE J. Selected Top. Signal Process 2(1), 57-73 (2008)

12. H Li, H Luo, G Jia, C Li, Dynamic resource allocation in OFDMA-based DF cooperative relay networks. Wireless Personal Commun. 62(3), 655-670 (2012)

13. S Kim, X Wang, M Madihian, Optimal resource allocation in multi-hop OFDMA wireless networks with cooperative relay. IEEE Trans. Wireless Commun. 7(5), 1833-1838 (2008)

14. LWeng, RD Murch, Cooperation strategies and resource allocations in multiuser OFDMA systems. IEEE Trans. Vehicular Technol. 58, 2331-2342 (2009)

15. M Salem, A Adinoyi, M Rahman, H Yanikomeroglu, D Falconer, Y Kim, Fairness-aware radio resource management in downlink OFDMA cellular relay networks. IEEE Trans. Wireless Commun. 9(5), 1628-1639 (2010)

16. M Mohamad, P Mary, M Helard, in IEEE Cross Layer Design (IWCLD), Third International Workshop on Cross Layer Design. Resource allocation for QoS aware relay-assisted OFDMA cellular networks (Rennes, Nov. 30 2011-Dec. 1 2011), pp. 1-6, 2011

17. B Hamed, SM Almodarresi, Dynamic resource allocation algorithm in multi-user cooperative OFDMA systems: considering QoS and fairness constraints. Wireless Netw. 18(4), 365-380 (2012)

18. M Awad, S Shen, in Proc. IEEE International Conference on Communications (ICC'08). OFDMA based two-hop cooperative relay network resources allocation (Beijing, May 19-23, 2008), pp. 4414-4418

19. D Zhang, Y Wang, J Lu, QoS aware relay selection and subcarrier allocation in cooperative OFDMA systems. IEEE Commun. Lett. 14(4), 294-296 (2010)

20. D Zhang, X Tao, J Lu, M Wang, Dynamic resource allocation for real-time services in cooperative OFDMA systems. IEEE Commun. Lett. 15(5), 497-499 (2010)

21. Z Han, T Himsoon, WP Siriwongpairat, KJR Liu, Resource allocation for multiuser cooperative OFDM networks: Who helps whom and how to cooperate. IEEE Trans. Vehicular Technol. 58(5), 2376-2391 (2009)

22. X Wang, GB Giannakis, AG Marques, A unified approach to QoS-guaranteed scheduling for channel-adaptive wireless networks. Proc. IEEE 95(12), 2410-2431 (2007)

23. T Quek, H Shin, M Win, Robust wireless relay networks: slow power allocation with guaranteed QoS. IEEE J. Selected Top. Signal Process $\mathbf{1}(4)$ 700-713 (2007)

24. DP Palomar, M Chiang, A tutorial on decomposition methods for network utility maximization. IEEE J. Selected Areas Commun. 24(8), 1439-1451 (2006)

25. DP Palomar, M Chiang, Alternative distributed algorithms for network utility maximization: framework and applications. IEEE Trans. Automatic Control 52(12), 2254-2269 (2007)

26. S Boyd, L Vandenberghe, Convex Optimization (Cambridge Univ. Press, Cambridge, 2004) 
27. M Grant, S Boyd, CVX:MATLAB Software for Disciplined Convex Programming (CVX Research, Inc, 2012). http://cvxr.com/cvx, Accessed 10 Jan 2013

28. DP Bertsekas, A Nedic, AE Ozdaglar, Convex Analysis and Optimization (Athena Scientific, Belmont, 2003)

29. R Jain, W Hawe, D Chiu, A Quantitative Measure of Fairness and Discrimination for Resource Allocation in Shared Computer Systems. DEC-TR-301 (ACM, New York, 1984)

Cite this article as: Zhang et al:: QoS-aware scheduling with optimization of base-station power allocation in downlink cooperative OFDMA systems. EURASIP Journal on Wireless Communications and Networking 2013 2013:247.

\section{Submit your manuscript to a SpringerOpen ${ }^{\odot}$ journal and benefit from:}

- Convenient online submission

- Rigorous peer review

- Immediate publication on acceptance

- Open access: articles freely available online

- High visibility within the field

- Retaining the copyright to your article

Submit your next manuscript at $\gg$ springeropen.com 